\title{
Clinical Classifiers to Identify Ascending Aortic Dilatation in Patients With Bicuspid Versus Tricuspid Aortic Valves
}

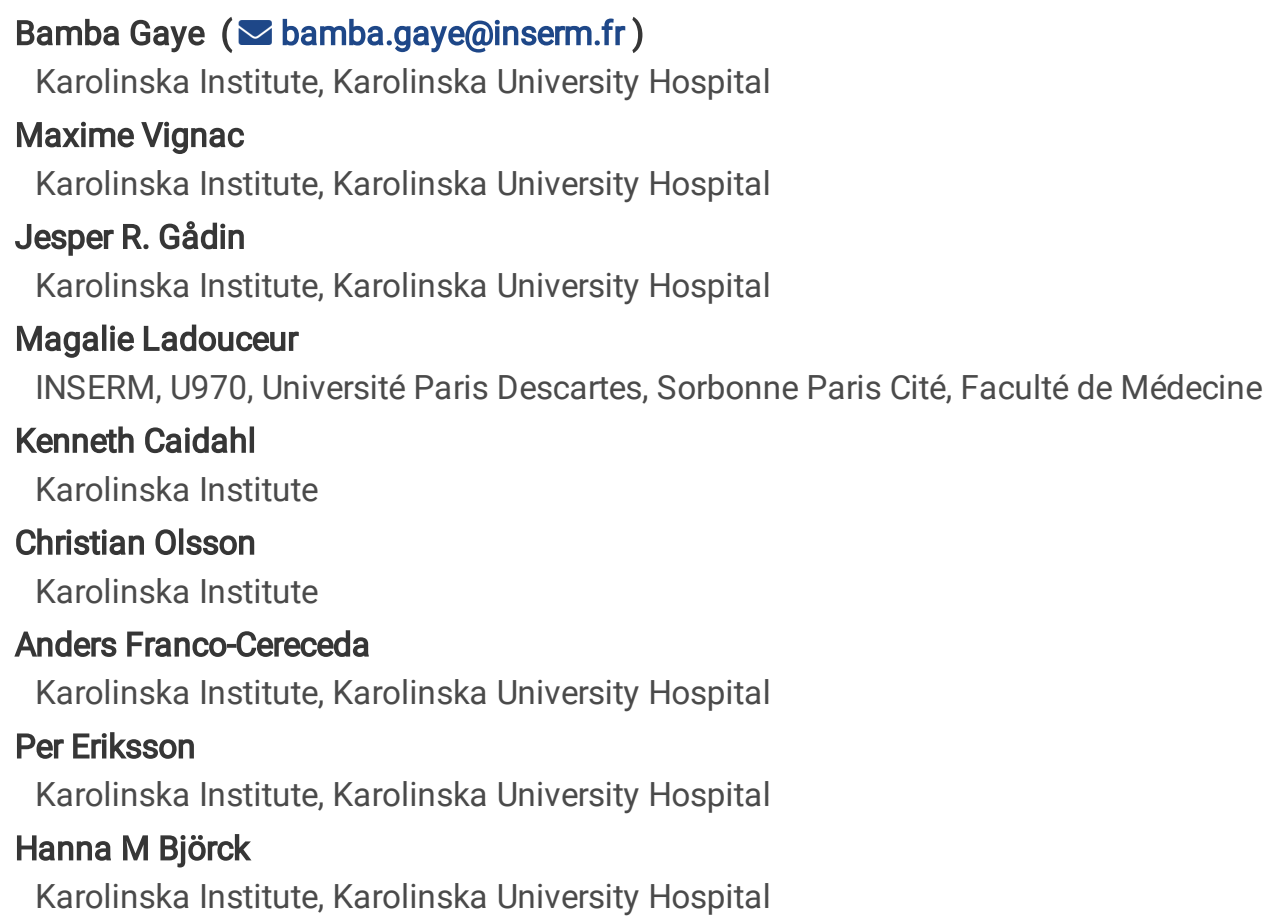

\section{Research Article}

Keywords: Bicuspid Aortic Valve, Tricuspid Aortic Valve, Ascending Aortic Aneurysm, Aortic Dilation

Posted Date: October 11th, 2021

DOl: https://doi.org/10.21203/rs.3.rs-957446/v1

License: (c) (i) This work is licensed under a Creative Commons Attribution 4.0 International License. Read Full License 


\section{Abstract}

Objective: We aimed to develop clinical classifiers to identify prevalent ascending aortic dilatation in patients with BAV and tricuspid aortic valve (TAV).

Methods: This study included BAV $(n=543)$ and TAV $(n=491)$ patients with aortic valve disease and/or ascending aortic dilatation but devoid of coronary artery disease undergoing cardiothoracic surgery. We applied machine learning algorithms and classic logistic regression models, using multiple variable selection methodologies to identify predictors of high risk of ascending aortic dilatation (ascending aorta with a diameter above $40 \mathrm{~mm}$ ). Analyses included comprehensive multidimensional data (i.e., valve morphology, clinical data, family history of cardiovascular diseases, prevalent diseases, demographic, lifestyle and medication).

Results: BAV patients were younger ( $60.4 \pm 12.4$ years) than TAV patients $(70.4 \pm 9.1$ years), and had a higher frequency of aortic dilatation ( $45.3 \%$ vs. $28.9 \%$ for BAV and TAV, respectively. $\mathrm{P}<0.001)$. The aneurysm prediction models showed mean AUC values above 0.8 for TAV patients, with the absence of aortic stenosis being the main predictor, followed by diabetes and high sensitivity CReactive Protein. Using the same clinical measures in BAV patients our prediction model resulted in AUC values between 0.5-0.55, not useful for prediction of aortic dilatation. The classification results were consistent for all machine learning algorithms and classic logistic regression models.

Conclusions: Cardiovascular risk profiles appear to be more predictive of aortopathy in TAV patients than in patients with BAV. This adds evidence to the fact that BAV- and TAV-associated aortopathy involve different pathways to aneurysm formation and highlights the need for specific aneurysm preventions in these patients. Further, our results highlight that machine learning approaches do not outperform classical prediction methods in addressing complex interactions and non-linear relations between variables.

\section{Key Questions}

\section{What is already known about this subject?}

Individuals with a bicuspid aortic valve (BAV), the most common congenital cardiac abnormality, are at higher risk of developing ascending aortic aneurysm than individuals born with a normal tricuspid aortic valve (TAV). ${ }^{1,2}$ Possible contributing factors to BAV is a turbulent flow, caused by the abnormal bicuspid valve anatomy, and/or a genetic defect.

\section{What does this study add?}

Neither the underlying pathophysiology nor a valid prediction model of the development of the aneurysm is available in patients with bicuspid aortic valves (BAV). Using automated machine learning algorithms and classic logistic regression models, we demonstrated that clinical predictors can predict ascending aortic dilatation in patients with tricuspid aortic valves (TAV) but not in patients with bicuspid aortic valves (BAV). Further, our results highlight that machine learning approaches do not outperform classical prediction methods in addressing complex interactions and non-linear relations between variables.

\section{How might this impact clinical practice?}

The lack of good models to develop clinical classifiers of BAV-associated ascending aortic dilatation strengthen the focus of genetics and/or flow as important contributing factors to aneurysm development in these individuals. Our study provides evidence to the fact that BAV- and TAV-associated aortopathy involve different pathways to aneurysm formation and highlights the need for specific aneurysm preventions in these patients.

\section{Introduction}

Thoracic aortic aneurysm (TAA) is a silent disease characterized by medial degradation and pathological widening of the intrathoracic aorta. Individuals with a bicuspid aortic valve (BAV) are at higher risk of developing ascending aortic aneurysm than individuals born with a normal tricuspid aortic valve (TAV). ${ }^{1,2} \mathrm{BAV}$ is the most common congenital cardiac abnormality with an incidence of about 13 per 1000 live births and more than one third of all BAV patients develop aneurysm or aortic dissection later in 
life. ${ }^{3,4}$ The underlying mechanism is not known although a turbulent flow, caused by the abnormal bicuspid valve anatomy, and/or a genetic defect have been proposed as contributing factors. The complications of BAV have led to a number of unanswered clinical questions such as: (1) what mechanisms are underlying the development of aortopathy in adults with BAV, (2) how to predict the development of aortopathy in individuals with BAV, and (3) will prediction models' performance and predictors be different in patients with BAV compared to patients with TAV. Echocardiography is effective to show the degree of the sinus Valsalva and the annulus dilatation. However, it is more difficult to know the degree of dilation of the sinotubular junction and the ascending aorta even though they are the more frequently dilatated sections in patients with BAV 5 .

The aim of the present study was to identify clinical classifiers of prevalent aortopathy using comprehensive multidimensional clinical data. Automated machine learning methods and traditional regression models was performed on a total of 1034 subjects with either BAV or TAV.

\section{Methods}

\section{Clinical cohorts}

The Advanced Study of Aortic Pathology (ASAP) study design and description have been previously published. ${ }^{6}$ Briefly, the ASAP cohort is a single-center, observational cohort study of consecutive patients with aortic valve and/or ascending aortic disease undergoing elective open-heart surgery at the Cardiothoracic Surgery Unit, Karolinska University Hospital in Stockholm, Sweden. Inclusion criteria were patients aged 18 or above with aortic valve disease (i.e., aortic stenosis; AS or aortic regurgitation; AR) and/or ascending aorta dilatation (aneurysm or ectasia) but devoid of significant coronary artery disease (stenosis $>70 \%$ or Fractional Flow Reserve (FFR) <0.80) and primarily not planned for another concomitant valve surgery. The Disease of the Aortic Valve Ascending Aorta and Coronary Arteries (DAVAACA) study was set up in the continuity of the ASAP study. The ASAP/DAVAACA study started in February 2007 and include multidimensional data (blood analyses, genetic and clinical data, family history of cardiovascular diseases, prevalent diseases data, demographic characteristics, lifestyle habits data and medication). In the present study, 1180 operated patients were included (June 28, 2017). Exclusion of patients with unicuspid aortic valves, patients with missing data for cuspidity and aortic dilatation and patients with syndromic forms of TAA resulted in a final study population of 1034 subjects (eFigure 1). Data collection and classification of AS and AR have been previously described. ${ }^{1}$ Aortic valve function and aortic dimensions were assessed according to the standards outlined by the American Society of Echocardiography. ${ }^{7-9}$ The study was approved by the Regional Ethics Review Board (application number 2006/784-31/1; 2012/1633-31/4), Stockholm, Sweden. Oral and written informed consent were obtained from all patients according to the declaration of Helsinki.

Preoperative transthoracic echocardiography (TTE) and transesophageal echocardiography (TEE) details as well as Aortic valve morphology determination are detailed in Supplemental Methods.

\section{Statistical analysis}

Characteristics of the population were described using ANOVA or Chi-square tests when appropriate. Several meaningful blocks of variables were chosen (clinical data, family history of cardiovascular diseases, prevalent diseases, demographic characteristics, lifestyle habits data and medication). Principal component analysis (PCA) and Multiple correspondence analysis (MCA) were used to explain the variance-covariance structure of the variables combined. To identify variables that separated dilated versus nondilated BAV and TAV patients, logistic regression models were used and automated machine learning algorithms were applied.

\section{Logistic Regression}

For logistic regression, a set of variables were selected to be included in the model. Since model performance can rely heavily on variable selection, different variable selection methods were tested prior to the logistic regression analysis. Firstly, variables were selected based on prior knowledge and/or biologic plausibility. Then, two automated variable selection methods were considered: (i) backward and forward elimination to optimize Akaike Information Criteria, (ii) and selection operator (LASSO).

Machine Learning Algorithms 
We used two machine learning algorithms, Random Forests and Artificial Neural Network, which are among the most widely and successfully used for clinical data. ${ }^{12}$ Each one of them represents a different algorithm "family", with different internal algorithm structures. ${ }^{13}$ Since it was not known beforehand which kind of algorithm would perform best, algorithms with different internal structures were chosen to increase the probability of good discriminative performance. A 10 -fold cross validation method to our logistic regression was also applied, allowing to split the training data into five subsets. Then, five times iteration over the five subsets were performed, such that the subset with the index same as the iteration number was used as the validation test and the remaining 4 subsets were training tests. Sensitivity, specificity, and positive and negative predictive values for each predictive model were also assessed, applying a leave one out cross validation method.

\section{Patient and Public Involvement}

Patients or the public were not involved in the design, or conduct, or reporting, or dissemination plans of our research.

\section{Results}

\section{Characteristics of BAV and TAV patients}

The study population included a total of 1034 patients (52\% BAV and $48 \%$ TAV) (e-Table 1, e-Figure 1). BAV patients were significantly younger (60.4 (SD 12.40) years vs. 70.4 (SD 9.09) years in TAV patients), and mainly men (73.1\%) compared to TAV patients (63.3\%). Furthermore, BAV patients had significantly higher body surface area (BSA) but, in contrast, a lower body mass index (BMI). AS was more common in BAV compared to TAV whereas no significant difference in the proportion of AR was found, even if the prevalence of AR was slightly higher in TAV patients. Finally, a higher prevalence of cardiovascular disease (CVD) history was observed in TAV individuals (hypertension, coronary artery disease, heart failure and stroke) and a higher percentage of family history of CVD (sibling history of myocardial infarction before 65 years of age).

\section{Aortic dilatation in BAV and TAV patients}

Globally, PCA showed no separation among BAV patients with (BAV-D) or without (BAV-ND) ascending aortic dilatation (Figure 1, Panel A), using general clinical characteristics, family history of CVD, prevalent diseases and demographics as input. By contrast, TAV patients with a dilated (TAV-D) ascending aorta clearly separated from TAV individuals with non-dilated (TAV-ND) ascending aortas (Figure 1, Panel B), indicating a more pronounced difference between these groups.

Analysis of patient descriptive data showed a pattern of significantly different associated traits between BAV-ND vs. BAV-D and TAVND vs. TAV-D (Table 1) patients. Specifically, TAV-D patients were younger than TAV-ND patients while in the BAV group age did not differ. BAV-D patients, on the other hand, had a higher BSA and diastolic blood pressure, and a lower pulse pressure (PP) than BAVND patients. In both BAV and TAV patients, AR was more commonly associated with aortic dilatation, whilst AS was more prevalent in patients with non-dilated aorta. It was also noted that aortic dilatation was more localized to the tubular part of the ascending aorta in BAV patients, whereas in TAV patients the dilatation more often included the aortic root (Table 1). A positive association between dilatation and abdominal aortic aneurysm (AAA), and negative associations between dilatation and diabetes, and dilatation and angina were found in both BAV and TAV patients (Table 1).

To further analyze BAV- and TAV-associated aortopathy, a multivariable logistic regression model was performed, including age, sex, BSA, low density lipoprotein (LDL) cholesterol and high sensitivity C-Reactive Protein (hs-CRP) as covariates (Table 2). Independent variables were chosen based on previous analyses with significant differences within groups. Covariates were selected based on stepwise selection and decision trees. The results showed that in BAV patients, AS, PP and diabetes were negatively associated with dilatation (adjusted models for age, sex, BSA, LDL and hsCRP were with AS: OR=0.44 (0.28-0.70), P=0.001; PP: OR=0.99 (0.98-1.0), $P=0.025$, and diabetes: $O R=0.32(0.16-0.62), P=0.001)$. Similarly, in TAV patients, dilatation was negatively associated with AS: $\mathrm{OR}=0.03(0.02-0.06), \mathrm{P}<0.001$ and diabetes $\mathrm{OR}=0.11(0.03-0.32), \mathrm{P}<0.001$. The magnitude of the association with AS was however 15-fold higher in TAV than in BAV patients. Aortic regurgitation was positively associated with aortic dilation in both BAV and TAV with a 5 -fold higher magnitude in TAV compared to BAV patients. (Table 2).

\section{Clinical classifiers of aortic dilatation}


In order to answer the question if the prevalence of aortopathy in TAV and BAV patients may be identified using clinical characteristics, automated machine learning algorithms, including Random Forests and Artificial Neural Network, and classic logistic regression models were used. This showed that the discrimination between TAV-ND and TAV-D patients was good for all models tested, with mean AUCs ranging from 0.81 to 0.88 (Figure 2, e-Table 2). The best discrimination was obtained using Random Forest (mean AUC: 0.88 (95\% Cl: 0.78-0.96)) and GLM (mean AUC: 0.81 (95\% Cl: 0.68-0.93)) (Figure 2, e-Table 2). Generalized Linear Regressions included AS, AR, PP, prevalence of diabetes, age, sex, BSA, LDL, hsCRP, BMI as variables. It was not possible to predict aortic dilatation in BAV individuals that are at high risk of developing aneurysm, as indicated by poor discrimination by all classification's models used (Figure 2, e-Table 2). This is in line with the lack of separation in the PCA.

The main contributor to the classifier in TAV was by far AS (e-Table 5). Accordingly, omitting AS as a covariate in the analysis between TAV patients with non-dilated and dilated aorta showed worse discrimination for all models (mean AUC: 0.704-0.729 for the different methods) (e-Figure 5). Other contributors included, among others, diabetes, AR and age. Of note, the robustness of our classification models is supported by the results of the sensitivity analyses using imputed data (e-Table 3, e-Table 4). Supplemental e-Table 4 indicates that the main characteristics between subjects with and without missing data did not differ substantially, minimizing selection bias.

\section{Discussion}

In our analysis of 1034 Swedish subjects with bicuspid and tricuspid aortic valves with and without prevalent aortopathy but devoid of coronary artery disease and primarily not planned for another concomitant valve surgery, we report two key findings. Firstly, prediction of aortic dilatation using automated machine learning methods and traditional regression models on multidimensional clinical data was possible only among TAV individuals, and not in patients with BAV. This suggests that general clinical cardiovascular risk profiles play more important roles during aortic dilation in TAV patients than in patients with a BAV, and further supports that aortopathy associated with BAV and TAV, respectively, are clearly distinct with different underlying etiologies. Secondly, our study shows that the classification results were consistent for all machine learning algorithms and classic logistic regression models. This suggest that machine learning approaches might not outperform classical prediction methods in addressing complex interactions and non-linear relations between variables.

The strongest predictor for ascending aortic dilatation in TAV was the absence of aortic stenosis. This is in accordance with our previous observations that surgical patients with AS and ascending aortic dilatation almost exclusively have a BAV ${ }^{14}$. Whether this imply that biological processes associated with the development of AS in TAV also contributes to ascending aortic stability needs to be further elucidated. AS is commonly caused by progressive calcification of the aortic valve and increase in prevalence with age. In our cohort, TAV patients with dilated ascending aortas were significantly younger than TAV patients with non-dilated ascending aortas, which may be one contributing factor to the observed association and possibly reflects the surgical nature of the cohort. However, omitting patients with AS from the analysis still resulted in significant predictive values, although with worse discrimination, indicating that age alone cannot explain the association. Other strong contributors to the prediction model were diabetes and hsCRP. A negative association between diabetes and aneurysm in the abdominal aorta is well documented. ${ }^{15}$ It has also been suggested that metformin prescription may associate with decreased risk of aortic dilatation and that the molecular mechanism involves a metformin-induced reduction in aortic inflammation. ${ }^{16,17}$ Similarly, an elevated hsCRP has been described in AAA patients and found to be an independent risk factor for AAA. ${ }^{18,19}$ Moreover, we have previously shown an inflammatory gene expression profile in dilated aortic tissue from TAV but not BAV patients. ${ }^{6}$ In the present study, there was a borderline significance of reduced hsCRP levels in BAV patients with dilated aorta. This together implies that aortic dilatation in TAV, in these aspects, may be more similar to aneurysm of the abdominal aorta than BAV aortic dilatation.

The lack of a good model for risk prediction of BAV-associated aortopathy raises the question, which other contributing factors may be of importance for aneurysm formation and development in these individuals? Two main hypotheses have been put forth in the literature.

Firstly, an altered flow in the proximal part of the ascending aorta due to the valve malformation itself has been suggested to provoke aortic dilatation. Also, different BAV morphotypes, i.e. cusp fusion pattern, have been shown to cause flow disturbances that affect the aorta in morphotype-dependent ways. ${ }^{18}$ In our study, flow characteristics were not included in the prediction model, which 
possibly could have influenced the results. However, we could not see any difference in the presence of aortic dilatation between different morphotypes, i.e. true BAV, LN-, RN- or RL cusp function (Table 1). Of note, other factors may also influence aortic flow patterns, e.g. eccentricity of valve opening due to valve disease, or vessel stiffness. Indeed, it has been suggested that AS significantly alters aortic hemodynamic and wall shear stress, independent of aortic valve phenotype.

Secondly, the genetic contribution may override the influence of traditional risk factors to aneurysm development in patients with BAV. Although specific gene(s) and/or mutation(s) underlying BAV and BAV-associated aortopathy are still to be unraveled, several genes have been shown to be associated with both BAV formation and concomitant aortopathy in mice and humans. ${ }^{19,20}$ Moreover, a high heritability of BAV and/or other cardiovascular malformations have been demonstrated using segregation patterns in families, with a heritability of BAV and BAV together with other cardiovascular malformations being as high as $89 \%$ and $75 \%$, respectively. ${ }^{21} \mathrm{~A}$ third, most likely, possibility is that both genetic factors and abnormal hemodynamic burden play central roles in BAV-associated aortopathy, interacting with each other and thereby contribute to aortic dilatation.

Further dissecting differences between patients with non-dilated and dilated ascending aortas we found that, among others, PP, AS, AR and diabetes were associated with dilatation in BAV. PP is a well-known risk factor for cardiovascular disease and the clinical manifestation of increased vascular stiffness. ${ }^{22}$ Surprisingly, PP was higher in BAV patients with a non-dilated aorta, which may seem counterintuitive since AR is associated with both increased PP and aortic dilatation. However, it may be speculated that the higher PP seen in these patients may rely on structural changes due to an increased hemodynamic burden associated with a BAV. In line with this, we have previously showed that BAV patients have a qualitative collagen defect in their ascending aorta, signified by a different collagen glycation compared with TAV patients and suggestive of an altered non-enzymatic collagen crosslinking. ${ }^{23}$ Interestingly, we have also shown that dilated ascending aorta of BAV patients display an increased collagen-related stiffness compared with TAV patients. ${ }^{24}$ Furthermore, The Strong Heart Study could also show that in patients free of prevalent coronary heart disease, aortic root dilatation was, at a given diastolic blood pressure and stroke volume, associated with lower pulse pressure. $^{25}$

Additionally, accelerated vascular aging and increased arterial stiffness has previously been described in diabetic patients, the proportion of which was higher among BAV patients with non-dilated aortas. ${ }^{26}$ However, we and others have previously demonstrated an increased vascular inflammation in dilated aorta in TAV- but not BAV patients, suggesting that other mechanisms could be involved in the protective role of diabetes on aneurysm formation in BAV patients. ${ }^{6,27}$ The association between dilatation and valve disease was not as pronounced in BAV- as in TAV patients, although AS was also negatively associated with dilatation in BAV, as previously found ${ }^{28}$ It may be speculated that the presence of AS increases flow velocities and blood pressure in the ascending aorta, thereby stimulating vascular remodeling and strengthening of the aortic wall. Whether this hamper the process of dilatation remains to be answered. The relation between degree of stenosis and width of the ascending aorta is complex, and a previous study found mid-ascending dilatation proportional to valve gradient when patients with small aortas were excluded. ${ }^{28}$

Our findings raise the issue of how to identify and implement prevention of aortopathy in BAV patients in a clinical setting. So far, clinicians have focused on aortic valve function and aortic dimensions to indicate cardiac surgery and recommend annual follow-up in asymptomatic patients to screen for associated aortopathy. ${ }^{29}$ High importance has been given to the morphology of the valve, although in our study, dilatation in BAV did not show any significant association with valve morphology. Of note, previous studies establishing an association between BAV cusp fusion and clinical outcomes relied on small sample size based on imaging diagnostic rather than anatomic diagnosis. ${ }^{30}$

\section{Study strengths and limitations}

In this study, comprehensive clinical data, including blood sampling as well as epidemiological data was used in the analysis of in total 1034 individuals (543 BAV and $491 \mathrm{TAV}$ ). The morphology of aortic valves was evaluated by visual inspection during openheart surgery, which is a major strength compared to only echocardiography in terms of reliability.

A few limitations must however be highlighted. Firstly, by design, only individuals devoid of significant coronary artery disease were included, which may introduce a selection bias and an overestimation of the prevalence of subjects with aneurysm. Secondly, as this is a population based surgical cohort, it is possible that our study population included BAV and TAV patients with worse 
outcomes compared to their counterparts of similar age. This should however not affect the associations between valve type and aortic dilatation. Lastly, TAV patients with non-dilated aortas were significantly older than TAV individuals with a dilated aorta, which could possibly explain the higher degree of patients with aortic stenosis in this group.

To conclude, using automated machine learning algorithms and classic logistic regression models we demonstrated that in TAV patients, cardiovascular risk profiles appear to be more predictive of aortopathy than in BAV patients. The good performance of the TAV classifier also after exclusion of AS offers important implications for better targeting TAV individuals that are of a high risk of developing aneurysm. The lack of good models to develop clinical classifiers of BAV-associated aortopathy strengthen the focus of genetics and/or flow as important contributing factors to aneurysm development in these individuals.

\section{Abbreviations}

AAA Abdominal Aortic Aneurysm

AR Aortic Regurgitation

AS Aortic Stenosis

ASAP Advanced Study of Aortic Pathology

BAV Bicuspid Aortic Valve

BMI Body Mass Index

BSA Body Surface Area

CVD Cardiovascular Disease

DAVAACA Disease of the Aortic Valve Ascending Aorta and Coronary Arteries

FFR Fractional Flow Reserve

Hs-CRP High Sensitivity C-Reactive Protein

LDL Low Density Lipoprotein

LN Left- and Non-Coronary Cusps

MCA Multiple Correspondence Analysis

PCA Principal Component analysis

PP Pulse Pressure

TAA Thoracic Aortic Aneurysm

TAV Tricuspid Aortic Valve

TEE Transesophageal Echocardiography

TTE Transthoracic Echocardiography

\section{Declarations}

\section{Acknowledgment:}

Author Contributions: Gaye, Franco-Cereceda, Eriksson, and Björck had full access to all the data in the study and take responsibility for the integrity of the data and the accuracy of the data analysis. 
Study concept and design: Gaye, Eriksson

Acquisition, analysis, or interpretation of data: Gaye, Vignac, Gådin, Ladouceur, Caidahl, Olsson, Franco-Cereceda, Eriksson, Björck

Drafting of the manuscript: Gaye

Critical revision of the manuscript for important intellectual content: Gaye, Vignac, Gådin, Ladouceur, Caidahl, Olsson, FrancoCereceda, Eriksson, Björck

Statistical analysis: Gaye and Vignac

Study supervision: Gaye, Eriksson

Funding: The work was supported by the Swedish Research Council [12660]; the Swedish Heart-Lung Foundation [20180451, 20170669]; the Stockholm County Council [20180072]; La Marató de TV3 [20151330]; and a donation by Fredrik Lundberg.

Disclosure: All authors have nothing to disclose.

Data Sharing: The ASAP/DAVAACA data are available for request from the Karolinska Institute (https://ki.se/) in Stockholm, Sweden and by contacting Hanna Björck (hanna.bjorck@ki.se).

\section{References}

1. Jackson, V. et al. Bicuspid aortic valve leaflet morphology in relation to aortic root morphology: a study of 300 patients undergoing open-heart surgery.Eur J Cardiothorac Surg. Published online May2011:S1010794011004623. doi:10.1016/j.ejcts.2011.04.014

2. Messner, B. \& Bernhard, D. Bicuspid aortic valve-associated aortopathy: Where do we stand? J Mol Cell Cardiol, 133, 76-85 https://doi.org/10.1016/j.yjmcc.2019.05.023 (2019).

3. Ward, C. Clinical significance of the bicuspid aortic valve., 83 (1), 81-85 https://doi.org/10.1136/heart.83.1.81 (2000).

4. Losenno, K. L., Goodman, R. L. \& Chu, M. W. A. Bicuspid Aortic Valve Disease and Ascending Aortic Aneurysms: Gaps in Knowledge. Cardiol Res Pract, 2012, 1-16 https://doi.org/10.1155/2012/145202 (2012).

5. Verma, S. \& Siu, S. C. Aortic dilatation in patients with bicuspid aortic valve. N Engl J Med, 370 (20), 1920-1929 https://doi.org/10.1056/NEJMra1207059 (2014).

6. Folkersen, L. et al. Unraveling Divergent Gene Expression Profiles in Bicuspid and Tricuspid Aortic Valve Patients with Thoracic Aortic Dilatation: The ASAP Study. Mol Med, 17 (11-12), 1365-1373 https://doi.org/10.2119/molmed.2011.00286 (2011).

7. Lang, R. M. et al. Recommendations for Chamber Quantification: A Report from the American Society of Echocardiography's Guidelines and Standards Committee and the Chamber Quantification Writing Group, Developed in Conjunction with the European Association of Echocardiography, a Branch of the European Society of Cardiology. J Am Soc Echocardiogr, 18 (12), 1440-1463 https://doi.org/10.1016/j.echo.2005.10.005 (2005).

8. Zoghbi, W. Recommendations for evaluation of the severity of native valvular regurgitation with two-dimensional and doppler echocardiography. J Am Soc Echocardiogr, 16 (7), 777-802 https://doi.org/10.1016/S0894-7317(03)00335-3 (2003).

9. Baumgartner, H. et al. Echocardiographic Assessment of Valve Stenosis: EAE/ASE Recommendations for Clinical Practice. J Am Soc Echocardiogr, 22 (1), 1-23 https://doi.org/10.1016/j.echo.2008.11.029 (2009).

10. Lambin, P. et al. Predicting outcomes in radiation oncology-multifactorial decision support systems. Nat Rev Clin Oncol, 10 (1), 27-40 https://doi.org/10.1038/nrclinonc.2012.196 (2013).

11. Fern'andez-Delgado, M., Cernadas, E., Barro, S. \& Amorim, D. Do we need hundreds of classifiers to solve real world classification problems? J Mach Learn Res. 2014;15(1).

12. Jackson, V. et al. Aortic dimensions in relation to bicuspid and tricuspid aortic valve pathology. J Heart Valve Dis, 23 (4), 463472 (2014).

13. Takagi, H., Umemoto, T. \& for the ALICE (All-Literature Investigation of Cardiovascular Evidence) Group. Negative association of diabetes with rupture of abdominal aortic aneurysm. Diab Vasc Dis Res, 13 (5), 341-347

Page 8/13 
https://doi.org/10.1177/1479164116651389 (2016).

14. Yu, X. et al. Metformin prescription and aortic aneurysm: systematic review and meta-analysis., 105 (17), 1351-1357 https://doi.org/10.1136/heartjnl-2018-314639 (2019).

15. Li, L., Mamputu, J-C., Wiernsperger, N. \& Renier, G. Signaling Pathways Involved in Human Vascular Smooth Muscle Cell Proliferation and Matrix Metalloproteinase-2 Expression Induced by Leptin: Inhibitory Effect of Metformin., 54 (7), $2227-2234$ https://doi.org/10.2337/diabetes.54.7.2227 (2005).

16. Vainas, T. et al. Serum C-Reactive Protein Level Is Associated With Abdominal Aortic Aneurysm Size and May Be Produced by Aneurysmal Tissue., 107 (8), 1103-1105 https://doi.org/10.1161/01.CIR.0000059938.95404.92 (2003).

17. Shangwei, Z. et al. Serum High-Sensitive C-Reactive Protein Level and CRP Genetic Polymorphisms Are Associated with Abdominal Aortic Aneurysm. Ann Vasc Surg, 45, 186-192 https://doi.org/10.1016/j.avsg.2017.05.024 (2017).

18. McNally, A., Madan, A. \& Sucosky, P. Morphotype-Dependent Flow Characteristics in Bicuspid Aortic Valve Ascending Aortas: A Benchtop Particle Image Velocimetry Study. Front Physiol, 8, https://doi.org/10.3389/fphys.2017.00044 (2017).

19. Yang, B. et al. Protein-altering and regulatory genetic variants near GATA4 implicated in bicuspid aortic valve. Nat Commun, 8 (1), 15481 https://doi.org/10.1038/ncomms15481 (2017).

20. Baylor-Hopkins Center for Mendelian Genomics et al. ROBO4 variants predispose individuals to bicuspid aortic valve and thoracic aortic aneurysm. Nat Genet, 51 (1), $42-50$ https://doi.org/10.1038/s41588-018-0265-y (2019).

21. Cripe, L., Andelfinger, G., Martin, L. J., Shooner, K. \& Benson, D. W. Bicuspid aortic valve is heritable. J Am Coll Cardiol, 44 (1), 138-143 https://doi.org/10.1016/j.jacc.2004.03.050 (2004).

22. Georgakis, M. K. et al. Genetically Predicted Blood Pressure Across the Lifespan: Differential Effects of Mean and Pulse Pressure on Stroke Risk., 76 (3), 953-961 https://doi.org/10.1161/HYPERTENSIONAHA.120.15136 (2020).

23. Wågsäter, D. et al. Impaired Collagen Biosynthesis and Cross-linking in Aorta of Patients With Bicuspid Aortic Valve. J Am Heart Assoc, 2 (1), https://doi.org/10.1161/JAHA.112.000034 (2013).

24. Forsell, C., Björck, H. M., Eriksson, P., Franco-Cereceda, A. \& Gasser, T. C. Biomechanical Properties of the Thoracic Aneurysmal Wall: Differences Between Bicuspid Aortic Valve and Tricuspid Aortic Valve Patients. Ann Thorac Surg, 98 (1), 65-71 https://doi.org/10.1016/j.athoracsur.2014.04.042 (2014).

25. de Simone, G. et al. Hemodynamic Correlates of Abnormal Aortic Root Dimension in an Adult Population: The Strong Heart Study. J Am Heart Assoc, 4 (10), https://doi.org/10.1161/JAHA.115.002309 (2015).

26. Schram, M. T. et al. Diabetes, pulse pressure and cardiovascular mortality: the Hoorn Study. J Hypertens, 20 (9), $1743-1751$ https://doi.org/10.1097/00004872-200209000-00017 (2002).

27. Grewal, N. et al. Ascending aorta dilation in association with bicuspid aortic valve: A maturation defect of the aortic wall. $J$ Thorac Cardiovasc Surg, 148 (4), 1583-1590 https://doi.org/10.1016/j.jtcvs.2014.01.027 (2014).

28. Della Corte, A. et al. Predictors of ascending aortic dilatation with bicuspid aortic valve: a wide spectrum of disease expression $\rrbracket$. Eur J Cardiothorac Surg, 31 (3), 397-405 https://doi.org/10.1016/j.ejcts.2006.12.006 (2007).

29. Baumgartner, H. et al. 2017 ESC/EACTS Guidelines for the management of valvular heart disease. Eur Heart J, 38 (36), $2739-$ 2791 https://doi.org/10.1093/eurheartj/ehx391 (2017).

30. Schaefer, B. M. et al. The bicuspid aortic valve: an integrated phenotypic classification of leaflet morphology and aortic root shape., 94 (12), 1634-1638 https://doi.org/10.1136/hrt.2007.132092 (2008).

\section{Tables}

Table 1. Baseline Characteristics of Patients with BAV or TAV (dilated vs. non-dilated ascending aorta). 


\begin{tabular}{|c|c|c|c|c|c|c|}
\hline & \multicolumn{3}{|c|}{ BAV patients $(n=543)$} & \multicolumn{3}{|c|}{ TAV patients $(n=491)$} \\
\hline & Dilated $(n=246)$ & $\begin{array}{l}\text { Non-Dilated } \\
(n=297)\end{array}$ & $\begin{array}{l}P \\
\text { value }\end{array}$ & $\begin{array}{l}\text { Dilated } \\
(n=142)\end{array}$ & $\begin{array}{l}\text { Non-Dilated } \\
(n=349)\end{array}$ & $\begin{array}{l}P \\
\text { value }\end{array}$ \\
\hline Gender: Male & $182(74.0 \%)$ & $215(72.4 \%)$ & 0.749 & $89(62.7 \%)$ & $222(63.6 \%)$ & 0.927 \\
\hline Age & $61.1(11.2)$ & $59.8(13.3)$ & 0.204 & $67.2(10.1)$ & $71.8(8.28)$ & $<0.001$ \\
\hline Height & $177(9.14)$ & $174(9.29)$ & 0.002 & $174(9.76)$ & $172(9.77)$ & 0.043 \\
\hline Weight & $83.1(15.6)$ & $80.9(15.8)$ & 0.100 & $81.4(16.3)$ & $81.6(15.7)$ & 0.909 \\
\hline BSA & $2.00(0.21)$ & $1.96(0.22)$ & 0.052 & $1.95(0.22)$ & $1.94(0.22)$ & 0.479 \\
\hline BMI & $26.5(4.15)$ & $26.6(4.36)$ & 0.916 & $26.9(4.41)$ & $27.7(4.90)$ & 0.087 \\
\hline Regular Smoker: & & & 0.176 & & & 0.537 \\
\hline No & $108(44.1 \%)$ & $142(48.8 \%)$ & & $61(43.3 \%)$ & $152(43.8 \%)$ & \\
\hline Former & $117(47.8 \%)$ & $117(40.2 \%)$ & & $69(48.9 \%)$ & $177(51.0 \%)$ & \\
\hline Yes & $20(8.16 \%)$ & $32(11.0 \%)$ & & $11(7.80 \%)$ & $18(5.19 \%)$ & \\
\hline Raphe: & & & 0.568 & & & \\
\hline True BAV & $24(9.96 \%)$ & $23(7.96 \%)$ & & & & \\
\hline Left-Non coronary & $1(0.41 \%)$ & $4(1.38 \%)$ & & & & \\
\hline Right-Non coronary & $43(17.8 \%)$ & $47(16.3 \%)$ & & & & \\
\hline Right-Left & $173(71.8 \%)$ & $215(74.4 \%)$ & & & & \\
\hline Systolic Blood Pressure & $135(18.2)$ & $136(18.9)$ & 0.299 & $144(17.4)$ & $142(21.5)$ & 0.299 \\
\hline $\begin{array}{l}\text { Diastolic Blood } \\
\text { Pressure }\end{array}$ & $81.3(12.3)$ & $79.0(11.6)$ & 0.026 & $75.1(13.0)$ & $76.5(13.3)$ & 0.290 \\
\hline Pulse Pressure (PP) & $53.3(16.5)$ & $57.2(18.3)$ & 0.009 & $68.7(18.1)$ & $65.4(20.3)$ & 0.076 \\
\hline Leukocytes & $5.70[4.70 ; 6.80]$ & $5.90[4.97 ; 7.00]$ & 0.052 & $\begin{array}{l}6.00 \\
{[5.00 ; 7.30]}\end{array}$ & $6.10[5.10 ; 7.40]$ & 0.386 \\
\hline hsCRP & $0.93[0.50 ; 2.15]$ & $1.20[0.59 ; 2.80]$ & 0.056 & $\begin{array}{l}2.10 \\
{[0.98 ; 4.05]}\end{array}$ & $1.60[0.71 ; 3.80]$ & 0.050 \\
\hline Cholesterol & $4.90[4.20 ; 5.70]$ & $4.80[4.20 ; 5.60]$ & 0.566 & $\begin{array}{l}4.80 \\
{[4.12 ; 5.40]}\end{array}$ & $4.60[3.90 ; 5.60]$ & 0.175 \\
\hline LDL & $2.90[2.20 ; 3.60]$ & $2.80[2.30 ; 3.60]$ & 0.813 & $\begin{array}{l}2.90 \\
{[2.20 ; 3.50]}\end{array}$ & $2.60[1.90 ; 3.40]$ & 0.064 \\
\hline Aortic Stenosis: & $169(68.7 \%)$ & $235(79.1 \%)$ & 0.008 & $23(16.2 \%)$ & $283(81.1 \%)$ & $<0.001$ \\
\hline Aortic Regurgitation: & $83(33.7 \%)$ & $75(25.3 \%)$ & 0.038 & $93(65.5 \%)$ & $67(19.2 \%)$ & $<0.001$ \\
\hline Diameter Aortic Anulus & $25.5(3.41)$ & $24.2(3.58)$ & $<0.001$ & $23.5(3.17)$ & $22.8(3.03)$ & 0.042 \\
\hline $\begin{array}{l}\text { Diameter Sinus } \\
\text { Valsalva }\end{array}$ & $38.1(5.38)$ & $33.6(5.11)$ & $<0.001$ & $40.1(6.98)$ & $32.8(5.71)$ & $<0.001$ \\
\hline Diameter STJ & $33.6(5.63)$ & $28.2(4.51)$ & $<0.001$ & $35.9(6.76)$ & $26.6(4.36)$ & $<0.001$ \\
\hline Diameter Ascend Aorta & $46.9(5.02)$ & $33.0(4.27)$ & $<0.001$ & $50.5(7.90)$ & $31.6(4.03)$ & $<0.001$ \\
\hline $\begin{array}{l}\text { Sibling MI before } 65 \\
\text { year }\end{array}$ & 13 (5.56\%) & 19 (6.81\%) & 0.688 & 11 (8.09\%) & 35 (10.8\%) & 0.474 \\
\hline $\begin{array}{l}\text { Mother MI before } 65 \\
\text { year }\end{array}$ & 15 (6.20\%) & 10 (3.64\%) & 0.250 & 7 (5.26\%) & 25 (7.67\%) & 0.474 \\
\hline
\end{tabular}




\begin{tabular}{|c|c|c|c|c|c|c|}
\hline & \multicolumn{3}{|c|}{ BAV patients $(n=543)$} & \multicolumn{3}{|c|}{ TAV patients $(n=491)$} \\
\hline & Dilated $(n=246)$ & $\begin{array}{l}\text { Non-Dilated } \\
(\mathrm{n}=297)\end{array}$ & $\begin{array}{l}P \\
\text { value }\end{array}$ & $\begin{array}{l}\text { Dilated } \\
(n=142)\end{array}$ & $\begin{array}{l}\text { Non-Dilated } \\
(\mathrm{n}=349)\end{array}$ & $\begin{array}{l}P \\
\text { value }\end{array}$ \\
\hline $\begin{array}{l}\text { Father MI before } 65 \\
\text { year }\end{array}$ & $24(10.6 \%)$ & $45(16.3 \%)$ & 0.087 & $18(14.1 \%)$ & $45(14.4 \%)$ & 0.922 \\
\hline Myocardial Infarction & $8(3.25 \%)$ & $11(3.77 \%)$ & 0.930 & $9(6.43 \%)$ & $33(9.46 \%)$ & 0.367 \\
\hline Stroke & $15(6.10 \%)$ & $15(5.08 \%)$ & 0.746 & $14(10.0 \%)$ & $43(12.3 \%)$ & 0.571 \\
\hline AAA & $23(9.39 \%)$ & $2(0.68 \%)$ & $<0.001$ & $25(18.2 \%)$ & $5(1.44 \%)$ & $<0.001$ \\
\hline Angina Pectoris & $9(3.66 \%)$ & $26(8.87 \%)$ & 0.023 & $9(6.43 \%)$ & $52(14.9 \%)$ & 0.015 \\
\hline Heart Failure & $15(6.15 \%)$ & $25(8.65 \%)$ & 0.354 & $15(10.6 \%)$ & $42(12.2 \%)$ & 0.747 \\
\hline Hypertension & $115(47.1 \%)$ & $140(47.6 \%)$ & 0.979 & $88(62.9 \%)$ & $223(65.0 \%)$ & 0.730 \\
\hline Diabetes & $15(6.10 \%)$ & $39(13.3 \%)$ & 0.009 & $3(2.16 \%)$ & $61(17.5 \%)$ & $<0.001$ \\
\hline ACE inhibitor & $46(18.7 \%)$ & $57(19.3 \%)$ & 0.941 & $45(31.9 \%)$ & $97(27.9 \%)$ & 0.434 \\
\hline Aspirin & $48(19.5 \%)$ & $84(28.5 \%)$ & 0.021 & $38(27.0 \%)$ & $155(44.8 \%)$ & 0.001 \\
\hline Betablocker & $92(37.4 \%)$ & 125 (42.4\%) & 0.277 & $76(53.9 \%)$ & $179(51.3 \%)$ & 0.672 \\
\hline
\end{tabular}

Chi square test was used for categorical variables; ANOVA for normal-distributed continuous variables; Kruskal-Wallis test for nonnormal continuous variables. BSA, body surface area; BMI, body mass index; hsCRP; high sensitive C-reactive protein; LDL, low density lipoprotein; STJ, sinotubular junction; AAA, abdominal aortic aneurysm.

Table 2. Predictors of ascending aortic dilatation in BAV and TAV patients, separately. Univariate and Multivariate logistic regression analysis.

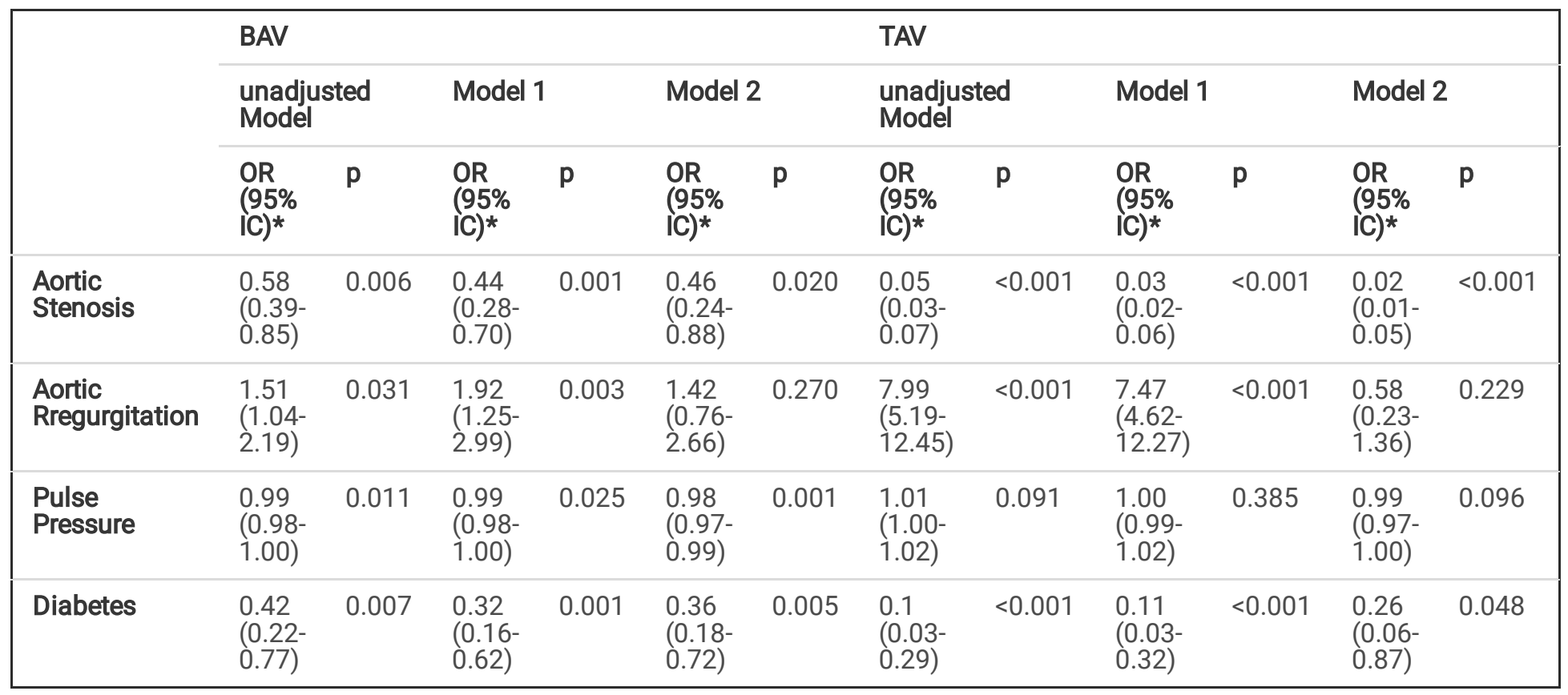

* Odds ratio and $95 \%$ confidence interval limits were obtained by logistic regression

Model 1: adjusted for Sex_Male + Age + BSA + LDL + hsCRP

Model 2: adjusted for Sex_Male + Age + BSA + LDL+ hsCRP + the 3 other variables (i.e: Aortic Rregurgitation, Pulse Pressure and Diabetes for Aortic Stenosis) 


\section{Figures}

Figure 1. Principal component analysis (PCA) plot of BAV versus TAV patients (with or without aneurysm).

\section{A, BAV patients}

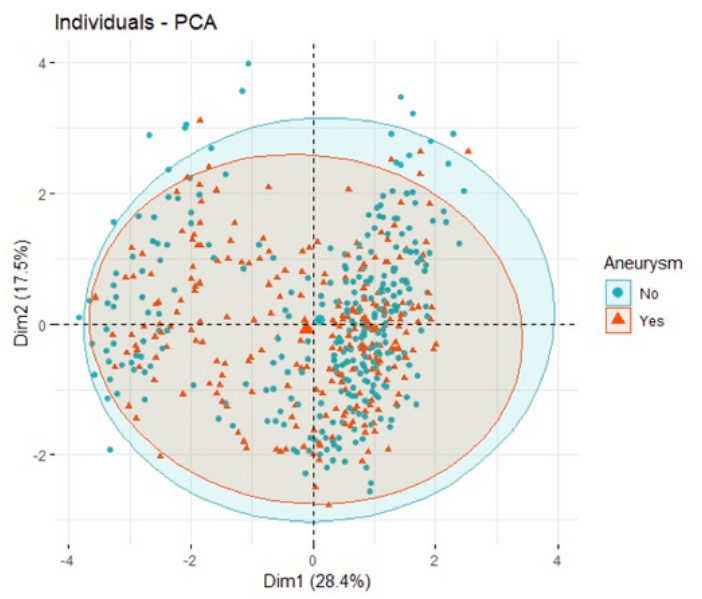

\section{B, TAV patients}

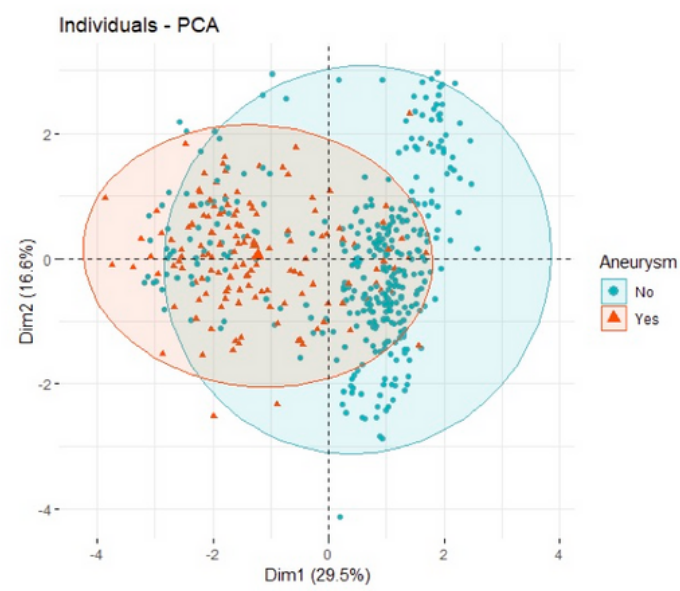

\section{Figure 1}

See image above for figure legend. 
Figure 2. The ROC curve for risk prediction model across the predictive methods used.
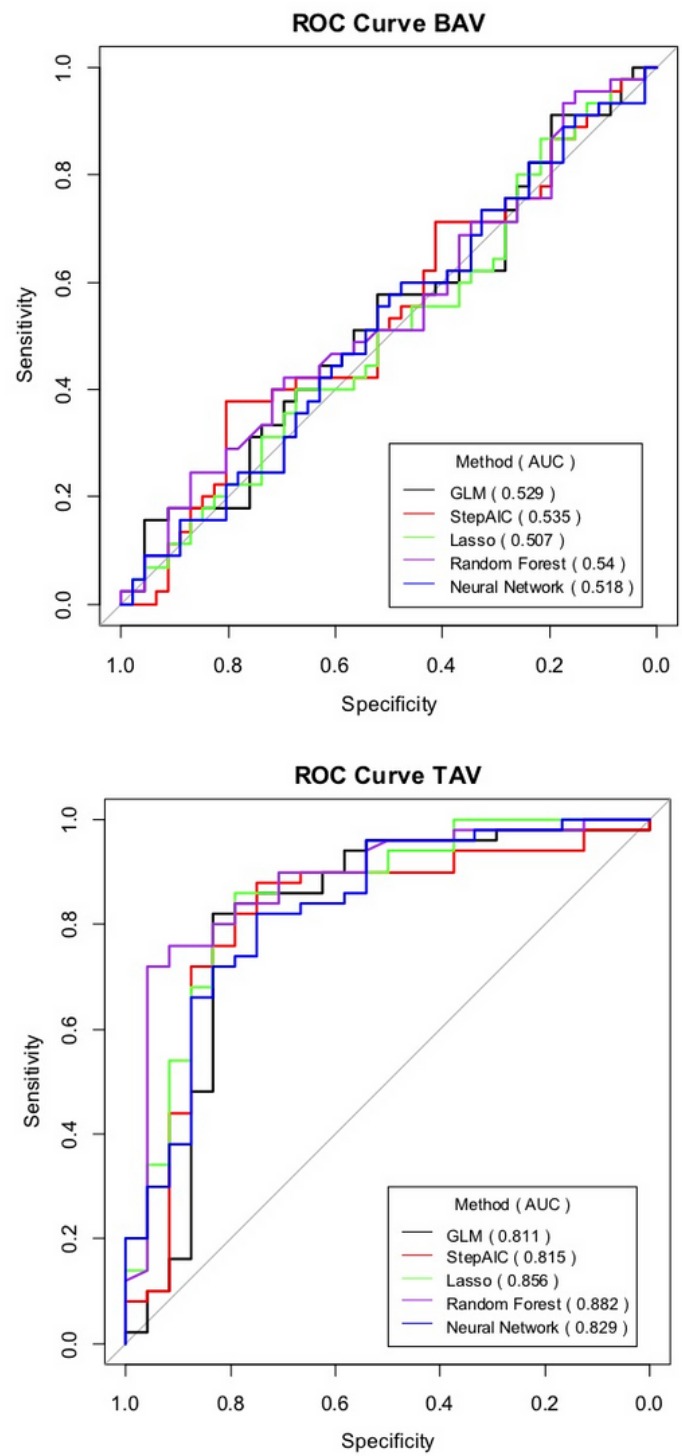

Figure 2

See image above for figure legend.

\section{Supplementary Files}

This is a list of supplementary files associated with this preprint. Click to download.

- Supplemental.pdf 